\title{
Refractory hypercalcaemia associated with disseminated Cryptococcus neoformans infection
}

\author{
Jasmine Jiang Zhu' ${ }^{1}$, William J Naughton ${ }^{2}$, Kim Hay Be ${ }^{3}$, Nicholas Ensor ${ }^{3}$ and \\ Ada S Cheung 1,4
}

1Department of Endocrinology, Austin Health, Victoria, Australia, 2Department of Infectious Diseases, Austin Health, Victoria, Australia, ${ }^{3}$ Liver Transplant Unit, Austin Health, Victoria, Australia, and ${ }^{4}$ Department of Medicine (Austin Health), The University of Melbourne, Victoria, Australia
Correspondence should be addressed to J J Zhu

Email

jasminejzhu@gmail.com

\section{Summary}

Hypercalcaemia is a very common endocrine condition, yet severe hypercalcaemia as a result of fungal infection is rarely described. There are have only been two reported cases in the literature of hypercalcaemia associated with Cryptococcus infection. Although the mechanism of hypercalcaemia in these infections is not clear, it has been suggested that it could be driven by the extra-renal production of 1-alpha-hydroxylase by macrophages in granulomas. We describe the case of a 55-year-old woman with a 1,25-OH D-mediated refractory hypercalcaemia in the context of a Cryptococcus neoformans infection. She required treatment with antifungals, pamidronate, calcitonin, denosumab and high-dose glucocorticoids. A disseminated fungal infection should be suspected in immunosuppressed individuals presenting with hypercalcaemia.

\section{Learning points}

- In immunocompromised patients with unexplained hypercalcaemia, fungal infections should be considered as the differential diagnoses;

- Glucocorticoids may be considered to treat 1,25-OH D-driven hypercalcaemia; however, the benefits of lowering the calcium need to be balanced against the risk of exacerbating an underlying infection;

- Fluconazole might be an effective therapy for both treatment of the hypercalcaemia by lowering 1,25-OH D levels as well as of the fungal infection.

\section{Background}

Hypercalcaemia is a very common endocrine condition, yet severe hypercalcaemia as a result of fungal infection is rarely described $(1,2,3,4)$.

\section{Case presentation}

A 55-year-old woman of Polynesian descent was presented 6 weeks after her second liver transplant with tachycardia and rising inflammatory markers. She had a history of end-stage liver disease due to non-alcoholic steatohepatitis cirrhosis, type 2 diabetes mellitus, obesity, hypertension and recurrent perianal abscesses. Her first liver transplant 4 months prior had failed as a result of acute rejection, hepatic bilomas, recurrent Enterococcus faecium and Pseudomonas aeruginosa bacteraemia and cytomegalovirus viraemia. She was immunosuppressed with cyclosporine $275 \mathrm{mg}$ and mycophenolate $1000 \mathrm{mg}$ twice daily. Her other medications included aspirin 100 mg daily, cholecalciferol 25 mcg daily, sulfamethoxazole/ trimethoprim 800/160 mg daily, enoxaparin $40 \mathrm{mg}$ daily, entecavir $0.5 \mathrm{mg}$ daily, valganciclovir $900 \mathrm{mg}$ daily, pregabalin $25 \mathrm{mg}$ twice daily and approximately 40 
units of basal-bolus insulin daily. She was not taking any thiazide diuretics.

A computed-tomography pulmonary angiogram revealed bilateral air space opacities (Fig. 1). Her Cryptococcus antigen titre was greater than 1 in 2560 . Culture of bronchial washings demonstrated the growth of Cryptococcus neoformans.

The patient was commenced on induction therapy with i.v. liposomal amphotericin $300 \mathrm{mg}$ daily and oral flucytosine $1 \mathrm{~g}$ twice daily. While on antifungal treatment, she developed invasive fungal skin lesions over her abdomen and thighs (Fig. 2) with biopsies demonstrating the growth of Cryptococcus neoformans. After 4 weeks, she transitioned to consolidation therapy with oral fluconazole $800 \mathrm{mg}$ daily. Ten days later, the patient developed further skin lesions, raising concerns about disease progression and she recommenced i.v. liposomal amphotericin and oral flucytosine at the former doses. A fluorodeoxyglucose PET (FDG-PET) scan demonstrated findings in keeping with disseminated cryptococcosis, with FDG-avid lesions in the lungs as well as substantial s.c. and muscle tissue avidity (Fig. 3).

Three weeks after her presentation, she was noted to have elevated corrected calcium of $2.79 \mathrm{mmol} / \mathrm{L}(2.10-2.60)$. Her calcium concentrations were observed and continued to rise steadily. The patient was surprisingly asymptomatic from the hypercalcaemia. She remained alert and oriented and did not experience polyuria or polydipsia. Throughout her hospitalisation she remained ambulant, mobile and had consumed a regular diet without any nutritional or calcium supplementation.

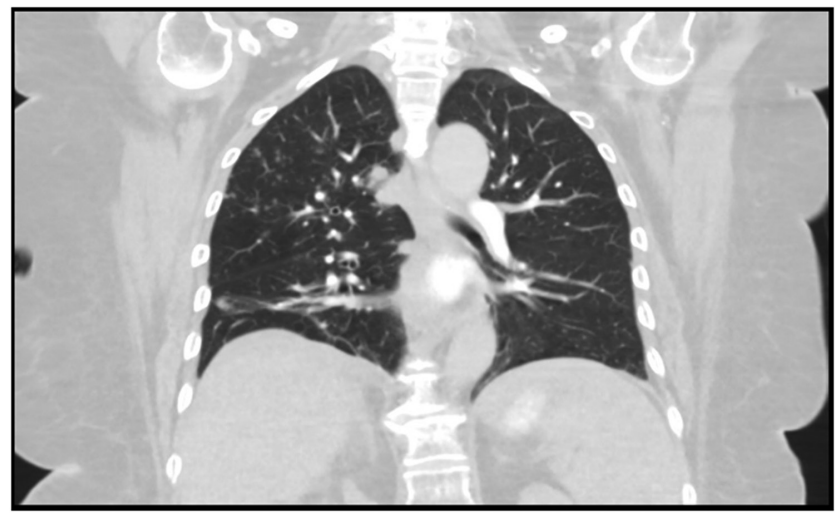

Figure 1

Bilateral scattered air space opacities demonstrated on a computertomography pulmonary angiogram.

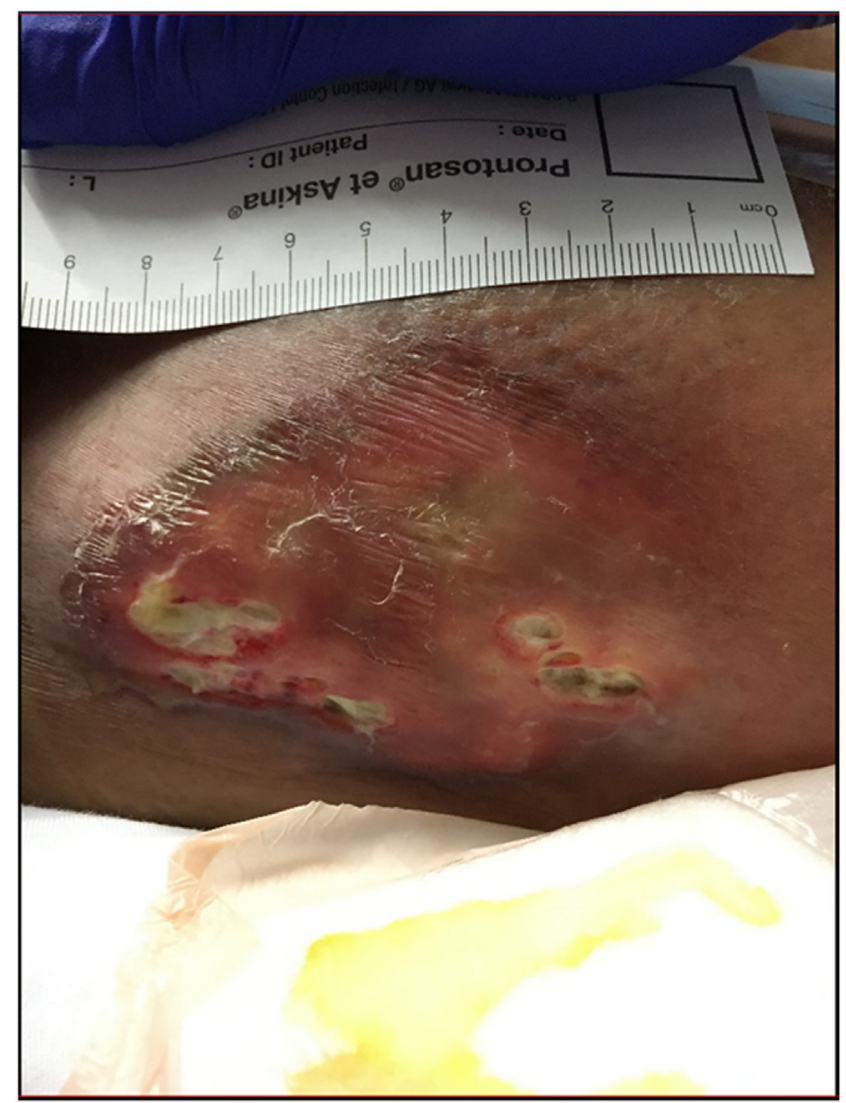

Figure 2

Cutaneous cryptococcosis with ulcerated pustular lesions.

\section{Investigation}

Further investigations when corrected calcium was 3.17 $\mathrm{mmol} / \mathrm{L}$ demonstrated that the ionised calcium was 1.69 $\mathrm{mmol} / \mathrm{L}(1.12-1.30)$, the parathyroid hormone $1.1 \mathrm{pmol} / \mathrm{L}$ (0.7-4.1), the thyroid stimulating hormone $4.96 \mathrm{mU} / \mathrm{L}$ (0.38-5.30), 25-hydroxy-vitamin D (25-OH D) $59 \mathrm{nmol} / \mathrm{L}$ and 1,25-dihydroxy-vitamin D (1,25-OH D) $219 \mathrm{pmol} / \mathrm{L}$ (50-190). The parathyroid hormone was measured on the Diasorin Liaison (1-84) assay which was subsequently found to report falsely high values at the lower end of the reference range. Her albumin was $35 \mathrm{~g} / \mathrm{L}$ (35-52) and the estimated glomerular filtration rate was $61 \mathrm{~mL} / \mathrm{min} / 1.73$ $\mathrm{m}^{2}(>90)$.

\section{Treatment}

As the cause of her hypercalcaemia was not immediately apparent, she was observed for several days before treatment with $30 \mathrm{mg}$ of i.v. pamidronate. Despite this, the corrected calcium continued to rise, peaking at $3.82 \mathrm{mmol} / \mathrm{L}$. The patient received a $48 \mathrm{~h}$ course of i.v. calcitonin $100 \mathrm{mg} 6 \mathrm{~h}$ followed by $60 \mathrm{mg}$ of s.c. denosumab. 


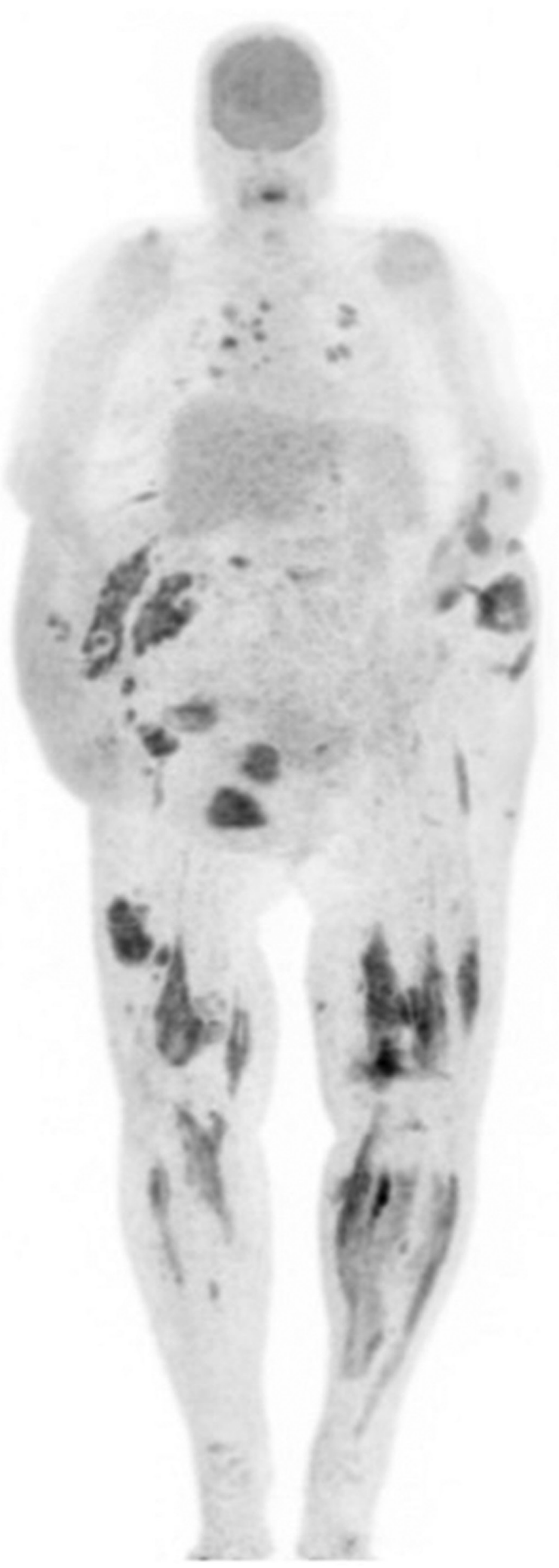

Figure 3

FDG-PET demonstrating FDG-avid lesions in s.c. and muscle tissues of forearms, lower torso and lower limbs, and FDG-avid pulmonary nodules and right hilar lymphadenopathy.

\section{Outcome and follow-up}

The calcium slowly decreased but remained above 3 $\mathrm{mmol} / \mathrm{L}$. Two weeks later, the patient had a liver biopsy demonstrating acute liver rejection and was treated with i.v. pulse methylprednisolone. The calcium levels sharply declined and normalised within several days (Fig. 4). There were no granulomas on the skin or liver biopsies.

She was discharged to the rehabilitation unit 4 weeks later and then was discharged home following a further 4 weeks. She remains normocalcaemic and continues on oral antifungals for an ongoing lower limb cutaneous Cryptococcus infection.

\section{Discussion}

Hypercalcaemia mediated by $1,25-\mathrm{OH} \mathrm{D}$ has been described as a rare complication of fungal infections. There are only two case reports in the literature of hypercalcaemia associated with Cryptococcus neoformans infection $(1,2)$. In both of these cases, the patients were infected with HIV and had a low CD4 lymphocyte count. Whilst the case described by Spindel had disseminated multiorgan Cryptococcus infection (1), Ali et al describe isolated pulmonary Cryptococcus neoformans and Coccidioides immitis infection (2). Both cases had an elevated 1,25-OH $\mathrm{D}$ and a peak corrected calcium in the range of 3.30-3.60 $\mathrm{mmol} / \mathrm{L}$. Hypercalcaemia was managed with i.v. fluids, antifungal treatment and one of the cases required i.v. pamidronate and hydrocortisone. Resolution of the hypercalcaemia coincided with the resolution of the Cryptococcus infection. Tuberculosis and lymphoma were excluded as alternative causes of hypercalcaemia.

Although the mechanism of hypercalcaemia in these infections is not clear, the fact that $1,25-\mathrm{OH} \mathrm{D}$ levels were elevated in all three cases suggest that the hypercalcaemia could be at least partly driven by the extra-renal production of 1-alpha-hydroxylase by macrophages in granulomas $(1,3)$. Indeed, Cryptococcus is known to induce a granulomatous response (5), and granulomas were found in the skin and liver biopsies of the case reported by Spindel (1). A review has suggested that $1,25-\mathrm{OH} \mathrm{D}$ may play a role in regulating immune function (4) so this pathway may become activated through adaptive mechanisms. However, there is no data to definitively support this theory.

Management of 1,25-OH D-driven hypercalcaemia includes the restriction of dietary calcium and sunlight exposure (4). Glucocorticoids inhibit 1-alpha-hydroxylase activity in macrophages as well as $1,25-\mathrm{OH}$ D-mediated absorption of calcium from the gastrointestinal tract, 


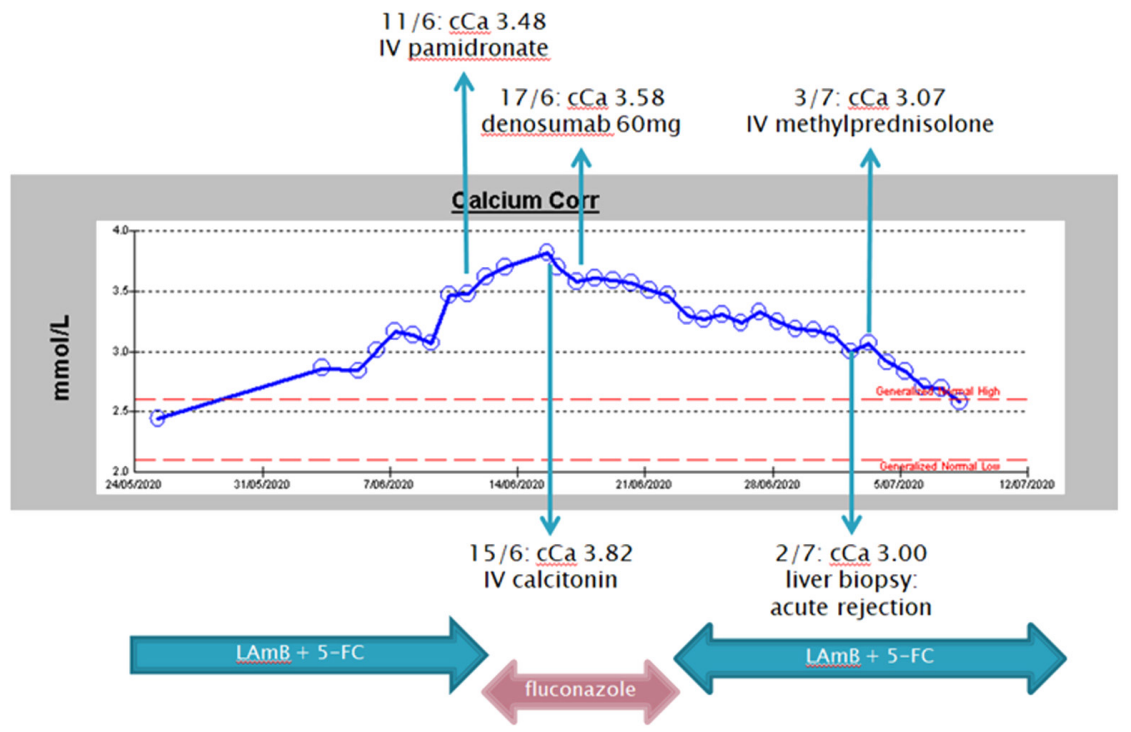

\section{Figure 4}

Trajectory of hypercalcaemia. The patient received initial treatment with i.v. liposomal amphotericin (LAmB) and oral flucytosine (5-FC). The calcium levels continued to rise following a pamidronate infusion, and then subsequently lowered after commencing a course of calcitonin followed by denosumab while on fluconazole. The hypercalcaemia resolved after methylprednisolone was administered for acute rejection of the liver graft. and may also be given. However, the risks of exacerbating an underlying infection need to be carefully considered against the benefit of controlling hypercalcaemia. In our case, methylprednisolone was administered as it was essential for the survival of the liver graft and fortuitously resolved the hypercalcaemia.

In addition to its antifungal effects, fluconazole inhibits 25-hydroxylase and 1-alpha-hydroxylase, and its use in reducing $1,25-\mathrm{OH} \mathrm{D}$ levels in a patient with a CYP24A1 mutation has been described (6). In our case, the calcium levels dropped during fluconazole therapy, but this may have been confounded by concurrent calcitonin and denosumab therapy.

In summary, Cryptococcus neoformans is a rare cause of 1,25-OH D-mediated refractory hypercalcaemia which in our case required antifungal treatment, pamidronate, calcitonin, denosumab and high dose glucocorticoid treatment. Hypercalcaemia is a rare complication of disseminated fungal infection, which should be suspected in immunosuppressed individuals.

\section{Declaration of interest}

The authors declare that there is no conflict of interest that could be perceived as prejudicing the impartiality of the research reported.

\section{Funding}

ASC is supported by a National Health and Medical Research Council of Australia Early Career Fellowship \#1143333.

\section{Patient consent}

Written consent has been obtained from the patient and is available upon request.

\section{Author contribution statement}

All authors were involved in the clinical care of the patient and contributed to the writing of the manuscript.

\section{References}

1 Spindel SJ, Hamill RJ, Georghiou PR, Lacke CE, Green LK \& Mallette LE 1995 Case report: vitamin D-mediated hypercalcemia in fungal infections. American Journal of the Medical Sciences 310 71-76. (https:// doi.org/10.1097/00000441-199508000-00007)

2 Ali MY, Gopal KV, Llerena LA \& Taylor HC 1999 Hypercalcemia associated with infection by Cryptococcus neoformans and Coccidioides immitis. American Journal of the Medical Sciences 318 419-423. (https://doi.org/10.1097/00000441-199912000-00010)

3 Lionakis MS, Samonis G \& Kontoyiannis DP 2008 Endocrine and metabolic manifestations of invasive fungal infections and systemic antifungal treatment. Mayo Clinic Proceedings 83 1046-1060. (https:// doi.org/10.4065/83.9.1046)

4 Sharma OP 2000 Hypercalcemia in granulomatous disorders: a clinical review. Current Opinion in Pulmonary Medicine 6 442-447. (https://doi. org/10.1097/00063198-200009000-00010)

5 Shibuya K, Hirata A, Omuta J, Sugamata M, Katori S, Saito N, Murata N, Morita A, Takahashi K, Hasegawa C, et al. 2005 Granuloma and cryptococcosis. Journal of Infection and Chemotherapy: Official Journal of the Japan Society of Chemotherapy 11 115-122. (https://doi. org/10.1007/s10156-005-0387-x)

6 Sayers J, Hynes AM, Srivastava S, Dowen F, Quinton R, Datta HK \& Sayer JA 2015 Successful treatment of hypercalcaemia associated with a CYP24A1 mutation with fluconazole. Clinical Kidney Journal 8 453-455. (https://doi.org/10.1093/ckj/sfv028)

Revised version received 10 November 2020 Accepted 10 May 2021 